1 Center for Outcomes Research and Evaluation, Yale New Haven Hospital, New Haven, Connecticut USA

2 Section of Cardiovascular Medicine, Department of Internal Medicine, Yale School of Medicine, New Haven, Connecticut USA

3 Department of Health Policy and Management, Yale School of Public Health, New Haven, Connecticut USA

4 Department of Pediatrics, Yale School of Medicine, New Haven, Connecticut USA

Twitter: @hmkyale, @DaisySMassey, @KarenSheares

Cite this as: BMJ 2022;376:0213 http://dx.doi.org/10.1136/bmj.o213 Published: 25 January 2022

\section{Racism as a leading cause of death in the United States}

\author{
Harlan M. Krumholz, ${ }^{1,2,3}$ Daisy S. Massey, ${ }^{1}$ Karen B. Dorsey ${ }^{1} 4$
}

During the past year, the dual crises of the covid-19 pandemic and police violence have opened many people's eyes to the ways in which the political construct of race-and anti-Black racism in particular-continue to determine who lives and who dies in the United States. Moreover, research is showing how little progress we are making in eliminating inequalities. ${ }^{1}$ Within medicine, physicians and other healthcare professionals are reckoning with the ways in which research has falsely looked at race as a biological attribute rather than a social construct over centuries, contributing to systems of racism in healthcare delivery. At long last, medical science is declaring that race is not biological, but that racism has profound consequences for health. ${ }^{2}$

To address racism, understand its impact on health, and identify and assess potential remedies, a national set of metrics is needed to galvanise action and promote accountability. During the pandemic, excess deaths have been used as a key metric to capture the full force of SARS-CoV-2 on the population. ${ }^{3}$ Timely, highly visible reporting of excess deaths under age 65 among Black people compared with White people-as a leading government health equity measure-could be a start. Kitagawa and Hauser noted the idea of excess mortality in $1974,{ }^{4}$ and it was part of the 1985 Report of the Secretary's Task Force on Black and Minority Health from the US.

Department of Health and Human Services. ${ }^{5}$

The current metrics related to leading causes of death focus attention on traditional clinical conditions, such as cardiovascular disease and cancer. Prevention, mitigation, and management of these diseases dominate medical training, and they consume a large portion of public and private financial resources in healthcare delivery and research. Each major disease typically has numerous public health organisations associated with promoting progress and supporting affected people, as well as specific months of the year dedicated to raising public awareness. Organisations and ordinary citizens raise millions of dollars to distribute to researchers who study these threats and search for cures. These combined efforts focus attention and galvanise action to reduce morbidity and mortality and improve overall health.

However, the risks of these diseases and others are mediated by a complex web of social determinants, which are acquired rather than innate. One of the most influential social determinants is systemic racism. Black people in the United States are more likely to die young-not because there is some intrinsic biological risk, but because of racism. ${ }^{26}$ The differences in life expectancy and mortality rates reflect the relationship between racism and health outcomes, which is complex and follows many potential pathways. This reality derives from the historical and present manifestation of racial politics in the United States, such as laws and policies that curtail individual freedoms, obstruct access to economic opportunities, and limit social mobility. For many racial and ethnic minority groups, and particularly for the descendants of enslaved Africans, equality in health and longevity remain beyond reach.

Excess deaths among Black people represent the difference over a discrete time between the number of deaths that occurred and the number of deaths that would have occurred had the mortality rate been the same as that among White people. The excess deaths associated with race can be understood as a toll that is in large part a result of racism in the United States. There is no biological reason, independent of social context, that Black people should die younger than White people. The excess premature deaths are the cumulative difference in death between Black and White people across every specific cause of death.

The excess deaths under age 65 by race can be put in context by comparing the toll with causes of death by each disease category. In sheer numbers, the excess deaths associated with Black race, that is the cumulative excess across each cause of death, are higher than the reported leading causes of death among Black men and women under age 65 , as indicated in a recently posted preprint..$^{8}$ The age-adjusted excess death rate was higher than the other causes each year from 1999 to 2019 among Black men and women under age 65 . While the excess death rate is lower than it was in 1999, it has been increasing since 2014 between Black and White men. In 2019, these differences produced 14444 (age-adjusted) excess deaths among Black women under age 65 and 25850 excess deaths among Black men under age 65 than would have occurred had their mortality risks been the same as those of White people. ${ }^{7}$ In contrast, in 2019, about 11500 Black women under age 65 died from malignant neoplasms and 9,900 died from diseases of the heart (age-adjusted). ${ }^{7}$ In 2019, about 17500 Black men under age 65 died from diseases of the heart and 11 800 died from malignant neoplasms (age-adjusted). ${ }^{7}$ These numbers do not yet account for the documented disparities in deaths due to covid-19, which have expanded the gap. ${ }^{10}$

The elimination of mortality disparities will require a holistic view of the root causes of individual and structural racism. ${ }^{6}$ Explicitly focusing national attention on the premature excess mortality related to race and racism may create a greater sense of urgency to solve this deeply rooted problem. The way public health data are presented and contextualised may be helpful in directing this attention and promoting societal accountability. We advocate for governments to publicly report excess deaths by race 
and ethnicity as a key health equity indicator and to be accountable for addressing the gaps.

Acknowledgment: The authors would like to thank Susannah M. Bernheim, (Center for Outcomes Research and Evaluation, Yale New Haven Hospital, New Haven, Connecticut; Section of General Internal Medicine, Yale School of Medicine, New Haven, Connecticut) and Yuan Lu, (Center for Outcomes Research and Evaluation, Yale New Haven Hospital, New Haven, Connecticut; Section of Cardiovascular Medicine, Department of Internal Medicine, Yale School of Medicine, New Haven, Connecticut) for their helpful comments on a prior version of the manuscript.

Competing interests: Karen Dorsey receives funding under contract with the Centers for Medicare and Medicaid Services to develop and maintain quality measures and from Humana, Inc. to advise on quality strategy. In the past three years, Harlan Krumholz received expenses and/or personal fees from UnitedHealth, IBM Watson Health, Element Science, Aetna, Facebook, the Siegfried and Jensen Law Firm, Arnold and Porter Law Firm, Martin/Baughman Law Firm, F-Prime, and the National Center for Cardiovascular Diseases in Beijing. He is a co-founder of Refactor Health and HugoHealth, and had grants and/or contracts from the Centers for Medicare and Medicaid Services, Agency for Healthcare Research and Quality, National Institutes of Health, American Heart Association, and Johnson and Johnson. Daisy Massey has no potential conflicts to report.

1 Mahajan S, Caraballo C, Lu Y, etal. Trends in Differences in Health Status and Health Care Access and Affordability by Race and Ethnicity in the United States, 1999-2018. JAMA2021;326:637-48. doi: 10.1001/jama.2021.9907 pmid: 34402830

2 Churchwell K, Elkind MSV, Benjamin RM, etalAmerican Heart Association. Call to Action: Structural Racism as a Fundamental Driver of Health Disparities: A Presidential Advisory From the American Heart Association. Circulation 2020;142:e454-68.

doi: 10.1161/CIR.0000000000000936 pmid: 33170755

3 Woolf SH, Chapman DA, Sabo RT, Weinberger DM, Hill L. Excess Deaths From COVID-19 and Other Causes, March-April 2020. JAMA 2020;324:510-3.

doi: 10.1001/jama.2020.11787 pmid: 32609307

4 Kitagawa EM, Hauser PM. Differential mortality in the United States. A study in socioeconomic epidemiology. Vital Health and Statistics Monographs, American Public Health Association, 1973. Available at: https://www.degruyter.com/document/doi/10.4159/harvard.9780674188471/html.

5 United States Department of Health and Human Services. Report of the Secretary's Task Force on Black and Minority Health. Washington, DC; 1985-86. Available at: https://collections.nlm.nih.gov/catalog/nlm:nlmuid-8602912-mvset.

6 Cogburn CD. Culture, Race, and Health: Implications for Racial Inequities and Population Health. Milbank Q 2019;97:736-61. doi: 10.1111/1468-0009.12411 pmid: 31512293

7 Centers for Disease Control and Prevention, National Center for Health Statistics, released in 2020. Data are from the Multiple Cause of Death Files, 1999-2019, as compiled from data provided by the 57 vital statistics jurisdictions through the Vital Statistics Cooperative Program.

8 Massey D, Faust J, Dorsey K, Lu Y, Krumholz H. Racism as a Leading Cause of Death: Measuring Excess Deaths in the US.medRxiv2021:2021.08.30.21262732doi: 10.1101/2021.08.30.21262732.

9 Egede LE, Walker RJ. Structural Racism, Social Risk Factors, and Covid-19 - A Dangerous Convergence for Black Americans. N Engl J Med 2020;383:e77. doi: 10.1056/NEJMp2023616 pmid: 32706952

10 Rossen LM. Disparities in Excess Mortality Associated with COVID-19-United States, 2020 MMWR Morb Mortal Wkly Rep 2021;•••:70doi: 10.15585/mmwr.mm7033a2. 